\title{
Capacidad reguladora de Chrysoperla externa (Hagen) sobre mosca blanca Trialeurodes vaporariorum (Westwood) en tomate bajo invernadero
}

\section{Chrysoperla externa (Hagen) regulatory capacity against whitefly Trialeurodes vaporariorum (Westwood) in tomato under glasshouse conditions}

Fecha de recepción: 1 de febrero de 2016

Mayerly Alejandra Castro-Lopez ${ }^{1}$ Fecha de aceptación: 7 de junio de 2016

\section{Resumen}

Trialeurodes vaporariorum Westwood (Hemíptera: Aleyrodidae) es uno de los principales limitantes del cultivo de tomate Solanum lycopersicum, por generar problemas directos e indirectos en este sistema de producción; para el manejo de este insecto (mosca blanca) se acude a excesivas aplicaciones de productos de síntesis química, sin obtener los mejores resultados. Este estudio consistió en evaluar, en condiciones de invernadero, la capacidad reguladora de Chrysoperla externa Hagen (Neuróptera: Chrysopidae) sobre la mosca blanca presente en tomate. En plantas infestadas con individuos de $T$. vaporariorum se adicionó C. externa en niveles poblacionales de 80, 160 y 240 individuos en estado larval por planta, y se contó con un testigo sin depredador. Se empleó un diseño completamente al azar en condiciones de invernadero (temperatura promedio de $20{ }^{\circ} \mathrm{C}$ y humedad relativa del $60 \%$ ). Se observaron diferencias estadísticamente significativas entre las densidades del depredador, con infestaciones de 35, 52 y $53 \%$ de T. vaporariorum (tras cuatro semanas), correspondientes a las liberaciones de 240, 160 y 80 individuos por planta, respectivamente. Se observó que las plantas del testigo presentaron menor altura y número de ramas, respecto de los tratamientos en los cuales había presencia del depredador C. externa.

Palabras clave: chrysopidae; control biológico; crisopas; entomófago; neuróptera.

1 Universidad Nacional de Colombia (Bogotá D.C., Colombia).

2 M.Sc. Universidad Pedagógica y Tecnológica de Colombia (Tunja, Colombia). john.martinez@uptc.edu.co. 


\begin{abstract}
Trialeurodes vaporariorum Westwood (Hemiptera: Aleyrodidae) is one of the main limiting factors disturbing tomato (Solanum lycopersicum) crop, due to its direct and indirect effects in the fruit's production system. This pest management has been focused on excessive chemical products, mostly without good results. This study aim was to evaluate under glasshouse conditions, the regulatory capacity of Chrysoperla externa Hagen (Neuroptera: Chrysopidae) against white fly in tomato. In tomato plants infested with $T$. vaporariorum, individuals of Chrysoperla externa were added at different levels, which consisted in 80, 160 and 240 individuals in larval stage per plant; also a control without the predator was used. To asses the experiment, a complete randomized design was used under glasshouse conditions (mean temperature of $20^{\circ} \mathrm{C}$ and relative humidity of $60 \%$ ). Significant statistical differences were observed between the predator densities with 35, 52 and 53 infestation percentages of $T$. vaporariorum (after 4 weeks), corresponding to the liberation of 240, 160 and 80 individuals per plant, respectively. Regarding with tomato, it was observed a lower plant height increase, plus smaller branches and less number per plant with the control, than the treatments with $C$. externa predator presence.
\end{abstract}

Keywords: biological control; chrysopidae; entomophagous; neuropteran; lacewings. 


\section{Introducción}

El tomate Solanum lycopersicum L. es una de las hortalizas más importantes a nivel mundial. En Colombia, según el Sistema de Estadísticas Agropecuarias -SEA-, en el periodo 2013-2014 su cultivo estuvo presente en 22 departamentos, con un área sembrada de 7.467 ha y una producción de 173.581 t; el departamento de Boyacá es el más productivo, con un área de 1.280 ha y una producción de $57.300 \mathrm{t}$, para un rendimiento de 92.6 t.ha-1. El sistema de producción de tomate se ve afectado por cambios climáticos, enfermedades e insectos-plaga, dentro de los cuales se destaca Trialeurodes vaporariorum (Westwood) (Hemíptera: Aleyrodidae) (1).

T. vaporariorum (mosca blanca) es uno de los grandes limitantes del cultivo de tomate, por ocasionar daños directos e indirectos en las plantas, como la perforación de las células del follaje, lo que impide el adecuado desarrollo fisiológico de estas; así mismo, este insecto excreta una sustancia azucarada, conocida como miel de rocío, que promueve el crecimiento del hongo Cladosporium pernum, que reduce la tasa fotosintética y causa la muerte de los tejidos (2); también se reporta a T. vaporariorum como vector de virus, dentro de los cuales se destacan Beet pseudoyellows virus y Tomato torrado virus $(3,4)$.

El manejo de la mosca blanca es muy complejo y se basa en estrategias químicas, pero este insecto presenta altos niveles de resistencia a insecticidas (5) como metamidofos (organofosforado), niveles de tolerancia a cipermetrina (piretroide) y niveles de susceptibilidad a metomil (carbamato) (6); así mismo, desarrolla biotipos, por su plasticidad genética, tiene capacidad de proliferación y amplio rango de plantas hospederas (7).

Debido a los problemas que han surgido en la actualidad con el uso de productos de síntesis química, ha surgido la necesidad de buscar alternativas que los sustituyan, como el uso de agentes biológicos. Son varios los artrópodos reportados como enemigos naturales de la mosca blanca, entre ellos se encuentran los Neurópteros, especialmente aquellos pertenecientes a la familia
Chrysopidae, que es una de las más grandes dentro de este orden, con cerca de 2000 especies descritas (8), de las cuales, Chrysoperla spp. se reconoce como un excelente depredador generalista, con un gran potencial de control biológico.

De acuerdo con Núñez (9), Chrysoperla externa (Hagen) se destaca por su amplio rango de depredación, adaptabilidad a diversos ambientes, alto índice para sobrevivir a la crianza en laboratorio y tolerancia a algunos insecticidas (10). Albuquerque et al. (11) reportan que este predador se alimenta agresivamente de 21 insectos-plaga de cultivos con importancia económica (12). Según Silva et al. (13), estos depredadores se han evaluado en laboratorio suministrándoles huevos y ninfas de mosca blanca, y han evidenciado una alta capacidad de depredación y potencial para reducir la densidad poblacional de esta (14). En estudios previos se evaluó la capacidad de depredación de C. externa sobre $T$. vaporariorum en condiciones de laboratorio, con porcentajes de mortalidad de $96.8 \%, 94.4 \%$ y $99.1 \%$ causados por el I, II y III instar larval, respectivamente (Castro et al. datos sin publicar); esto indica que C. externa constituye una herramienta potencial de control biológico para su implementación en el manejo de T. vaporariorum para el cultivo de tomate.

Con el fin de complementar los estudios realizados en laboratorio, el objetivo del presente trabajo consistió en evaluar la capacidad reguladora del estado larval de $C$. externa sobre mosca blanca $T$. vaporariorum, en condiciones de invernadero, con el fin de determinar si a través de la interacción depredador-presa se puede proyectar el uso de este controlador en el manejo integrado de esta plaga en condiciones bajo cubierta.

\section{Materiales y métodos}

\section{A. Localización geográfica del área de estudio}

La investigación se llevó a cabo en el invernadero del Grupo de Manejo Biológico de Cultivos (GMBC), ubicado en la Universidad Pedagógica y Tecnológica de Colombia (UPTC), en el municipio de Tunja, departamento de Boyacá, Colombia; bajo 
una temperatura promedio de $20{ }^{\circ} \mathrm{C}$ y humedad relativa del $60 \%$.

\section{B. Procedimiento experimental}

Se realizó la siembra de 50 plántulas de tomate Solanum lycopersicum L., tipo chonto, en invernadero, previamente adquiridas en el municipio de Sáchica (Boyacá). Las plantas se confinaron en jaulas de $1.5 \mathrm{~m}$ de altura, elaboradas con tela tul y armazón de madera; cada plántula de tomate se infestó con material vegetal (hojas) con presencia de individuos de mosca blanca $T$. vaporariorum, adicionado dentro de las jaulas. Se empleó un manejo convencional relacionado con el riego, la fertilización y demás labores agronómicas, para lograr un buen desarrollo de las plantas hasta inicio de la floración.

\section{Evaluación del efecto de la liberación de Chrysoperla externa}

Cuando se presentó una alta infestación (100\%) de mosca blanca $T$. vaporariorum, evidenciada por la presencia de todos los estados de desarrollo de la plaga, se liberaron diferentes cantidades del depredador para las pruebas. Los tratamientos fueron de 80 (T1), 160 (T2) y 240 (T3) individuos de C. externa por planta, y un testigo sin aplicación del depredador. Cada repetición estuvo constituida por una planta de tomate. Dichos depredadores fueron adquiridos en Laboratorios Perkins Ltda., que los comercializa en estado de huevo con cascarilla de arroz como sustrato de soporte, para su liberación una vez inicia la emergencia de las larvas.

Se realizó un primer muestreo 24 horas después de la liberación, y se efectuaron conteos diarios, con el fin de verificar la presencia de la especie depredadora y su establecimiento y supervivencia en las plantas.
El efecto regulador se evaluó estableciendo la presencia de ninfas de I, II y III instar de $T$. vaporariorum, por planta tratada, en el $25 \%$ del follaje presente, de acuerdo con el desarrollo de la planta mediante observaciones diarias. Así mismo, se evaluó el crecimiento de las plantas de tomate a través de las variables de altura y número de ramas.

El diseño estadístico empleado fue completamente al azar (DCA), con 4 tratamientos y 10 repeticiones por tratamiento, para un total de 40 unidades experimentales (UE). Los resultados se analizaron con el programa SAS (SAS, 2001). Se usó un análisis de varianza (Anova) para detectar diferencias entre tratamientos y comparación de medias utilizando la prueba de Tukey, con un nivel de significancia de $P=0.05$.

\section{Resultados y discusión}

\section{A. Evaluación del efecto de la liberación de Chrysoperla externa}

El $100 \%$ de infestación de T. vaporariorum con diferentes poblaciones de individuos en estado larval de C. externa sobre plantas de tomate presentó diferencias significativas entre los tratamientos evaluados. El tratamiento T3 (240 individuos C. externa) se diferenció de los tratamientos T1 y T2 (80 y 160 individuos, respectivamente) $(\mathrm{P}<0.05)$; se aplicó la prueba de Tukey y se estableció que se presentan así dos grupos estadísticamente diferentes: uno constituido por T3, y otro, por T1 y T2 (Figura 1). El testigo absoluto (T) no se incluyó en el análisis, debido a que no se presentó mortalidad en $T$. vaporariorum, concordante con la ausencia del depredador, manteniendo una infestación constante del $100 \%$ de los individuos de mosca blanca. 


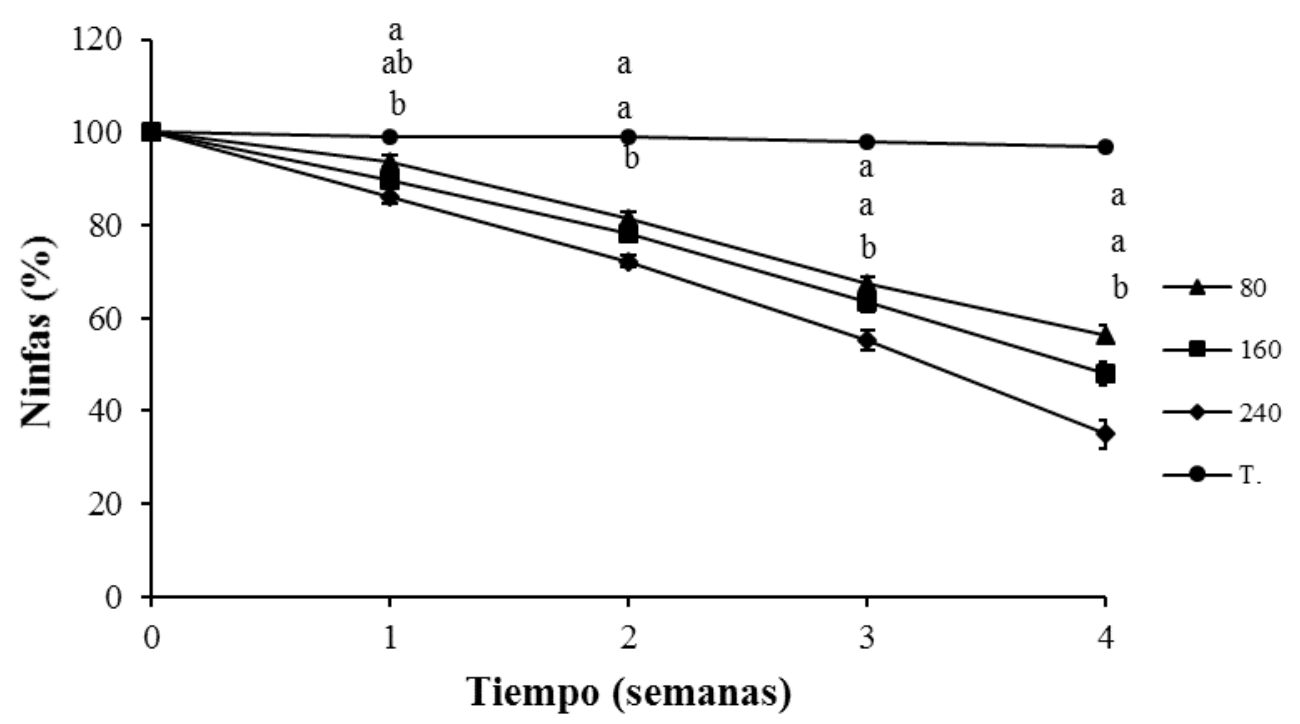

Fig. 1. Variación en el porcentaje de infestación de individuos de T. vaporariorum por el efecto regulador del estado larval de C. externa durante el tiempo de evaluación.

Se observó, de forma general, que las densidades de individuos de $T$. vaporariorum presentes en las plantas de tomate decrecieron a medida que transcurrió el tiempo en todos los tratamientos evaluados. El T3 fue el más efectivo, dado que finalizadas las 4 semanas de evaluación se presentó $35 \%$ de infestación de estados ninfales de mosca blanca en relación con el testigo en las plantas de tomate, con un porcentaje de control del $65 \%$ (Fig. 1); este resultado es similar al obtenido por Auad et al. (15), con una reducción cercana al 50 $\%$ de ninfas de Bemicia tabaci por consumo de $C$. externa.

Se constató mayor control de la capacidad reguladora de $C$. externa en la última semana del ensayo (Figura 1); esto se puede explicar teniendo en cuenta que el depredador se encuentra en el III instar larval, y por ello presenta mayor tamaño y mayor movilidad, lo que favorece un área de búsqueda mayor, incrementando el número de presas consumidas, como lo verifican diferentes autores, comoSchlick-Souza et al. (16) con presas de Aphis gossypii, (Hemíptera: Aphididae) y Bastidas et al. (17) con individuos de Neohydatothrips signifer (Thysanoptera: Thripidae).
En los muestreos realizados posliberación (PL) se verificó mayor consumo del tercer estadio ninfal de T. vaporariorum; este comportamiento se atribuye al tamaño, ya que al ser más grandes las ninfas de la presa, mayor es la eficiencia de búsqueda del depredador; los instar larvales de C. externa prefieren este estado y no otros, por la dificultad para encontrar, por ejemplo, los huevos de mosca blanca, como mencionan Auad et al. (15). Sin embargo, se recomienda que la liberación de este depredador se realice cuando se presenten densidades bajas de la presa, porque este puede aumentar la búsqueda y eficiencia de manipulación, pudiendo gastar un poco más de tiempo con cada presa, logrando extraer el suficiente contenido nutricional, tal como lo afirman Landeros et al. (18) en su evaluación de Chrysoperla carnea sobre huevos de $T$. vaporariorum.

Con el fin de lograr mayor eficiencia reguladora con C. externa, se debe liberar cuando está en su primer instar larval y cuando los niveles de infestación de la plaga sean de bajos a moderados, como lo afirma Monje (19), quien obtuvo un porcentaje de mortalidad del $76 \%$ de la plaga $N$. signifer en el cultivo de maracuyá durante todo el estadio larval del depredador C. externa. 
Se observó desarrollo normal y supervivencia del estado larval de $C$. externa con un promedio de temperatura de $20{ }^{\circ} \mathrm{C}$ y humedad relativa de 60 $\%$, bajo condiciones de invernadero; valores que se encuentran dentro de los rangos expuestos por Alves et al. (20) y Oliveira et al. (21), quienes indican que temperaturas de $16{ }^{\circ} \mathrm{C}$ a $28{ }^{\circ} \mathrm{C}$ permiten un desarrollo satisfactorio de las fases inmaduras de este depredador, y que valores de $12{ }^{\circ} \mathrm{C}$ y $32{ }^{\circ} \mathrm{C}$ presentan un bajo porcentaje de supervivencia, afectando la implementación de este controlador biológico.

Lo anterior indica que C. externa puede sobrevivir en un rango relativamente amplio de temperaturas y que podría ser empleado en las zonas o cultivos que se encuentren dentro de estos valores. Estas condiciones ambientales permitieron el desarrollo normal del estado larval del depredador, pudiéndose observar su duración y los cambios morfológicos que este presentaba.

\section{B. Efecto de la presencia del depredador en el desarrollo de las plantas}

La altura de las plantas de tomate $(\mathrm{en} \mathrm{cm})$, respecto a las diferentes cantidades de producto comercial de C. externa evaluados, presentó diferencias significativas entre los tratamientos y el testigo absoluto, pero no entre los tratamientos; esto fue corroborado posteriormente con la prueba de Tukey $(\mathrm{P}<0.05)$.

En la Figura 2 se observa que las plantas con los tratamientos T1, T2 y T3 presentaron una altura de 110,109 y $115 \mathrm{~cm}$, respectivamente, mientras que las del testigo alcanzaron una altura de solo $80 \mathrm{~cm}$, lo cual se puede encontrar directamente relacionado con el efecto del depredador, que fue el único factor diferencial.

Lo anterior se explicaría porque las plantas con presencia de adultos de $T$. vaporariorum presentaron una reducción de la turgencia de las hojas, lo cual afecta el sistema vascular, reduciendo la capacidad de acumular fotoasimilados para el crecimiento y el mantenimiento de la planta (4); sin embargo, estos valores fueron menores que los expuestos por Bernal et al. (1), que obtuvieron una altura de $140 \mathrm{~cm}$ en la semana 16 después de la siembra; esta diferencia se debe, posiblemente, a la labor de manejo agronómico que realizaron los autores, denominada deshoje, que en el presente ensayo no fue realizada, y que pudo influir en la distribución espacial de las ninfas de mosca blanca.

La fase reproductiva del tomate se presentó, aproximadamente, a las ocho semanas después de la siembra, coincidiendo con lo reportado por Jaramillo et al. (22).

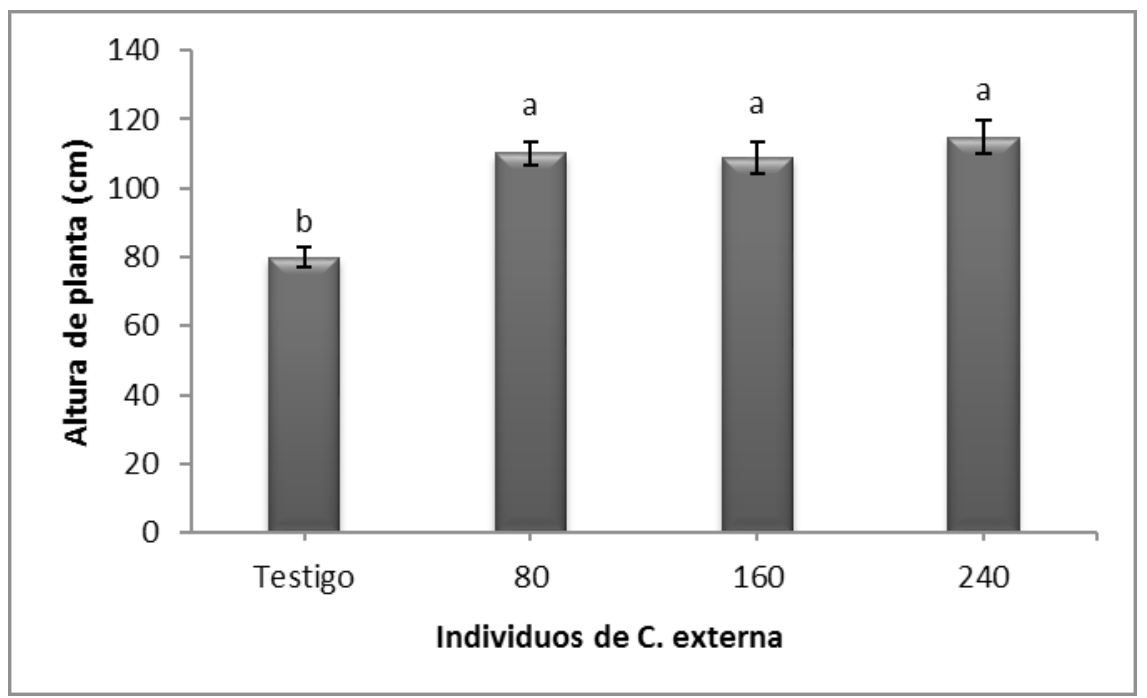

Fig. 2. Altura de la planta $(\mathrm{cm})$ respecto a las diferentes poblaciones $C$. externa evaluadas, expresadas como número de individuos aplicados por planta. 
Cuando se presenta un ataque por mosca blanca, las plantas no reconocen la acción de esta, debido a que este fitófago evita activar los mecanismos de defensa, utilizando un canal salivar que le permite entrar entre los tejidos de la planta sin producir daño mecánico. Igualmente, se ha registrado que en la fisionomía del aparato bucal de este insectoplaga se presenta un órgano que le permite regular la cantidad de fluido que entra por presión al estilete (23), afectando así el desarrollo normal de la planta.
En cuanto al número de ramas emitidas por las plantas de tomate (Figura 3), se determinó que, de la misma forma, la presencia de individuos de C. externa originó diferencias significativas entre los tratamientos evaluados y el testigo absoluto (sin depredador), pero no entre los tratamientos, corroboradas con la prueba de Tukey $(\mathrm{P}<0.05)$.

Los valores registrados en este estudio, de un promedio de 13 ramas por planta en los tres tratamientos evaluados y de 9 ramas en el testigo absoluto, se encuentran dentro del rango obtenido por Bernal et al. (1).

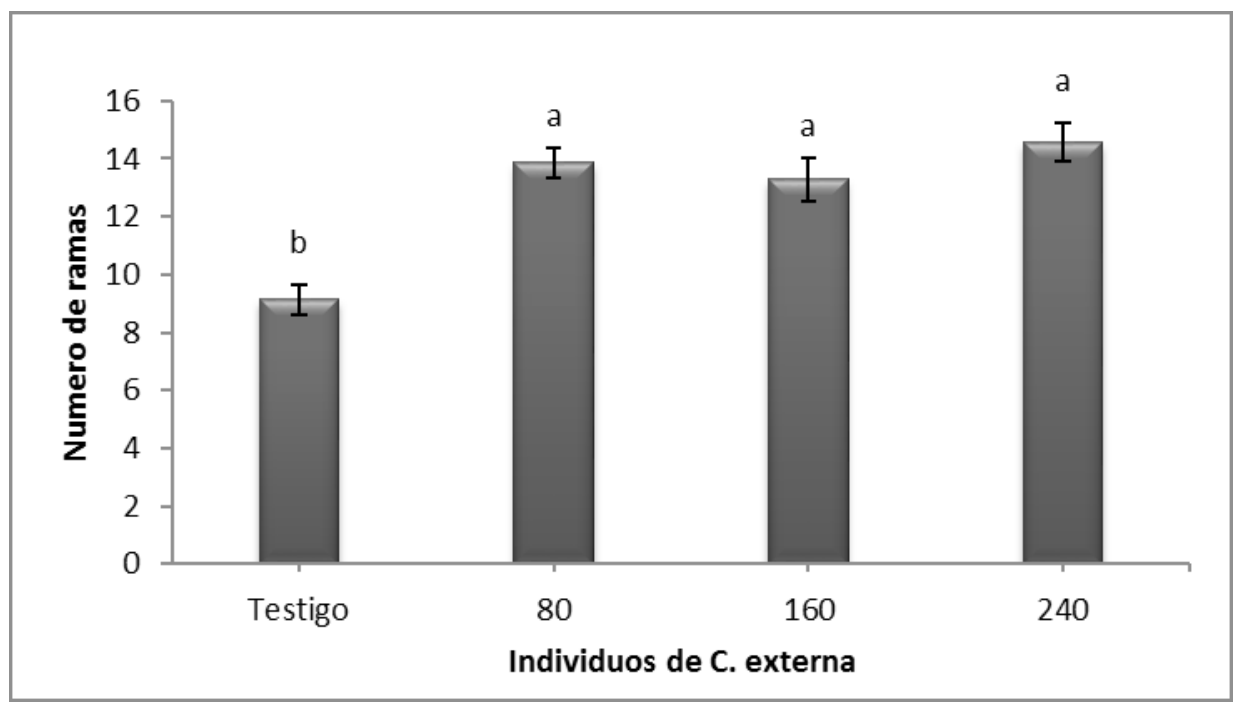

Fig. 3. Numero de ramas en plantas de tomate respecto a las diferentes poblaciones de C. externa expresadas como número de individuos aplicados por planta.

Como se ilustra en las Figuras 2 y 3, a pesar de no presentarse diferencias estadísticamente significativas entre los tratamientos, sí se observó que en el T3 (240 individuos de C. externa) se presentaron mayores valores respecto a altura y número de ramas, siendo nuevamente la mayor acción reguladora del insecto depredador en dicho tratamiento (por la mayor cantidad de individuos liberados) sobre la plaga $T$. vaporariorum, la que genera este efecto. El menor crecimiento del testigo, por lo tanto, se da porque $T$. vaporariorum presenta una constante alimentación para cubrir sus requerimientos nutricionales, extrayendo gran cantidad de fluido del floema, que es rico en aminoácidos y azucares para la planta (5) lo cual afecta el desarrollo normal de las plantas.

En general, también se puede considerar que la capacidad de control se puede ver afectada cuando se presenta alta densidad de la plaga, debido que se produce gran cantidad de miel de rocío, cuando este fitófago, después de insertar su estilete en el tejido de la planta, permanece alimentándose y excretando los productos de la digestión continuamente en el día; lo anterior puede afectar la movilidad y la capacidad de búsqueda del depredador para hallar su presa, como se observó en el estudio realizado por Pérez (24), donde por esta razón se vio afectado el porcentaje de 
parasitismo de Encarsia formosa (Hymenoptera: Aphelinidae) sobre ninfas de T. vaporariorum.

Finalmente, en condiciones naturales no se debe descartar la presencia en los cultivos de este importante agente de control biológico, como son las crisopas, por presentarse diversas especies en el neotrópico (13), como lo demuestran Pinho et al. (25), que asociaron la presencia de especies como Chrysoperla externa, Ceraeochrysa cubana, Ceraeochrysa cincta, Ceraeochrysa sp., Chrysopodes elongatus y Chrysopodes sp. en cultivos de tomate en las ciudades Cambuci y Seropédica, en el estado de Rio de Janeiro, Brasil. Lo anterior es posible si en el sistema de producción de tomate se realizan ajustes tecnológicos que permitan reducir el impacto de los agroquímicos en las poblaciones de benéficos nativos.

\section{Conclusiones}

El tratamiento T3 (240 individuos del depredador por planta) fue el más efectivo; dando como resultado un control del $65 \%$, es decir, la infestación de ninfas de Trialeurodes vaporariorum fue de solo el $35 \%$ al finalizar el ensayo.

La altura y el número de ramas de Solanum lycopersicum $L$. fueron mayores en los tratamientos con presencia de Chrysoperla externa, en comparación con el testigo absoluto, en donde la mayor presencia de individuos de Trialeurodes vaporariorum afectó claramente el desarrollo de las plantas.

Chrysoperla externa (Hagen) puede ser considerada una herramienta potencial de control biológico para su implementación en el manejo de Trialeurodes vaporariorum (westwood) en el cultivo de tomate bajo cubierta en Boyacá, aunque se requieren algunas pruebas bajo un sistema de producción comercial para su validación.

\section{Agradecimientos}

A la Dirección de Investigaciones de la Universidad Pedagógica y Tecnológica de Colombia, por la cofinanciación de este proyecto (SGI 1733) y a los demás integrantes del Grupo Manejo Biológico de Cultivos.

\section{Referencias}

(1) Bernal L, Pesca L, Rodríguez D, Cantor F, Cure J. Plan de muestreo directo para Trialeurodes vaporariorum (Westwood) (Hemíptera: Aleyrodidae) en cultivos comerciales de tomate. Agronomía Colombiana. 2008; 26(2): 266276.

(2) Gullan PJ, Cranston PS. The Insects: An Outline of Entomology. 4th ed. Chichester, UK: John Wiley \& Sons. 2010.

(3) Jones DR. Plant Viruses Transmitted by Whiteflies. Eur J Plant Pathol. 2003; 109(3): 195-219. DOI: http://dx.doi.org/10.1023/A:1022846630513.

(4) Suárez L, Díaz M, Rodríguez D, Cantor F. Medición indirecta de la tasa de consumo de adultos e inmaduros de Trialeurodes vaporariorum (Hemíptera: Aleyrodidae) sobre fríjol. Acta Biol. Colomb. 2015; 20(3): 99-109. DOI: http://dx.doi.org/10.15446/abc.v20n3.44073.

(5) Cardona C, Rendón F, García J, López-Ávila A, Bueno J, Ramírez J. Resistencia a insecticidas en Bemisia tabaci y Trialeurodes vaporariorum (Homoptera: Aleyrodidae) en Colombia y Ecuador. Revista Colombiana de Entomología. 2001; 27: 33-38.

(6) García J, Benítez E, López A. Efecto de la densidad de población de Trialeurodes vaporariorum (Hemíptera: Aleyrodidae) sobre la eficiencia del depredador Delphastus pusillus (Coleoptera: Coccinellidae). Revista Corpoica Ciencia y Tecnología Agropecuaria, 2007; 8(2): 17-21.

(7) Van Lenteren JC. A greenhouse without pesticides: fact or fantasy? Crop Protection. 2000; 19: 375-384. DOI: http://dx.doi.org/10.1016/ S0261-2194(00)00038-7. 
(8) Salamanca J, Varón E, Santos O. Cría y evaluación de la capacidad de depredación de Chrysoperla externa sobre Neohydatothrips signifer, trips plaga del cultivo de maracuyá. Revista Corpoica Ciencia y Tecnología Agropecuaria. 2010; 11(1): 31-40.

(9) Núñez F. Ciclo biológico y crianza de Chrysoperla externa y Careochrysa cinta (Neuróptera: Chrysopidae). Revista Peruana de Entomología. 1998; 31: 76-82.

(10) Pineda M. Efectividad biológica del depredador Chrysoperla carnea Stephens en el control de plagas. Tesis de grado Ingeniero Agrónomo Horticultor, Facultad de Ciencias Agropecuarias, Universidad Michoacana de San Nicolás de Hidalgo. Michoacán, 2007; 79 p.

(11) Albuquerque G, Tauber C, Tauber M. Chrysoperla externa (Neuroptera: Chrysopidae): Life history and potential for biological control in Central and South. 1994; 4(1): 8-13.

(12) Cadena P, Ángel F, Gómez L, González R. Diferenciación morfológica y molecular de especies de Crisópidos (Neuróptera: Chrysopidae). Revista Colombiana de Entomología. 2007; 33(2): 171-177.

(13) Silva P, Albuquerque G, Tauber C, Tauber M. Life history of a widespread Neotropical predator, Chrysopodes (Chrysopodes) lineafrons (Neuroptera: Chrysopidae). Biological Control. 2007; 41(1): 33-41. DOI: http://dx.doi. org/10.1016/j.biocontrol.2006.12.004.

(14) Adriano E, Toscano L, Schlick E, Itamar M, Lemes F. Desenvolvimento e capacidade de consumo de Chrysoperla externa (Hagen, 1861) alimentada com ninfas de mosca-branca criadas em hortaliças. Revista Caatinga. 2010; 23(3): 1-6.

(15) Auad M, Carvalho C, Souza B, Simões A, Oliveira S, Furtado A, Botelho R. Potencial de Chrysoperla externa (Hagen) no controle de Bemisia tabaci (Gennadius) biótipo B em tomateiro. Acta Scientiarum. Agronomy. 2007;
29(1): 29-32. DOI: http://dx.doi.org/10.4025/ actasciagron.v29i1.62.

(16) Schlick-souza E, Toscano L, Souza-schlick G, Maruyama W, Andrade A. Desenvolvimiento larval de Chrysoperla externa alimentada com Aphis gossypii provenientes de três cultivares de algodoeiro. Revista Agrarian Dourados. 2011; 4(13): 182-188.

(17) Bastidas J, Devia D, Santos O. Cría y evaluación de la capacidad de depredación de Chrysoperla externa sobre Neohydatothrips signifer, trips plaga del cultivo de maracuyá. Revista Corpoica Ciencia y Tecnología Agropecuaria. 2010; 11(1): 31- 40.

(18) Landeros J, Guevara L, Aguirre L, Cerna E, Badii M, Ochoa Y. Respuesta Funcional de Chrysoperla carnea (Neuroptera: Chrysopidae) sobre Huevos de Trialeurodes vaporariorum (Hemiptera: Aleyrodidae). Southwestern Entomologist. 2013; 38(2): 345-352. DOI: http:// dx.doi.org/10.3958/059.038.0217.

(19) Monje B. Componentes para el manejo integrado de Neohydatothrips signifer priesner 1932. (Thysanoptera: Thripidae) en maracuyá amarillo (Passiflora edulis degener) var. flavicarpa, en el departamento del Huila. Tesis de grado Maestría en Ciencias Agrarias-Entomología, Facultad de Ciencias, Universidad Nacional de Colombia. Medellín, 2012; 118.

(20) Alves S, Souza B, Machado A. Aspectos biológicos y capacidad depredadora de Chrysoperla externa (Neuróptera: Chrysopidae) alimentada con Sipha flava (Hemiptera: Aphididae). En: Memorias, XXXV Congreso Sociedad Colombiana de Entomología (SOCOLEN), Cali, Colombia. 2008; 80-81.

(21) Oliveira S, Auad A, Souza B, Silva D, Carvalho $C$. Effect of temperature on the interaction between Chrysoperla externa (Neuróptera: Chrysopidae) and Sipha flava (Hemiptera: Aphididae). Eur. J. Entomol. 2010; 107: 183188. DOI: http://dx.doi.org/10.14411/ eje.2010.024. 
(22) Jaramillo J, Rodríguez M, Guzmán M, Zapata T. Manual técnico: buenas prácticas agrícolas en la producción de tomate bajo condiciones protegidas. FAO, Gobernación de Antioquia, FAO-mana, Corpoica, Rionegro, 2007.

(23) Stansly PA, Naranjo SE. Bemisia: Bionomics and Management of a Global Pest. Dordrecht: Springer Netherlands; 2010. DOI: http:// dx.doi.org/10.1007/978-90-481-2460-2.

(24) Pérez D. Efecto del parasitoide Encarsia formosa (hymenoptera: aphelinidae) sobre una población de Trialeurodes vaporariorum (westwood) (Hemíptera: Aleyrodidae) en un cultivo comercial de tomate (Lycopersicon esculentum, Mill) bajo condiciones de invernadero. Universidad Militar Nueva Granada. Bogotá, Colombia, 2008.

(25) Pinho A, Guimarães J, Félix da Costa R, Torres P. Species of Chrysopidae (Neuroptera) associated to trellised tomato crops in two cities of Rio de Janeiro State, Brazil. Agricultural Entomology. 2015; 82: 1-4. 